\title{
Optimal Rate and Power Adaptation for Multirate CDMA
}

\author{
Syed Ali Jafar and Andrea Goldsmith \\ Stanford University \\ Wireless Systems Laboratory \\ Packard Electrical Engineering, \\ \{syed, andrea\}@wsl.stanford.edu
}

\begin{abstract}
We determine the optimal centralized adaptive rate and power control strategy to maximize the total average weighted throughput in a generic multirate CDMA system with slow fading. An average power and instantaneous bit error rate (BER) constraint is assumed at the transmitter with conventional matched filter detection at the receiver. Our results are general enough to apply to several multirate CDMA schemes: multi-code, multi-processing gain, multirate modulation or hybrids of these. We obtain compact polyhedral set representations for the optimal rate regions without assuming any symmetries between the users. Moreover we show that as the users move around within the cell, i.e. as the propagation path loss and the distribution of the channel fading of each user changes, the optimal power and rate control strategy can adapt by simply scaling the channel fades of the users. Our numerical results show that increasing the granularity of rate adaptation (i.e. increasing the number of intermediate rate levels) for the same range of rates (i.e. the same maximum and minimum rate) does not significantly increase the average throughput.
\end{abstract}

\section{Introduction}

The importance of adaptation to improve the spectral efficiency of a wireless communication system is well recognized. Rate and power adaptation strategies have been explored under the assumption of perfect channel state information at the transmitter and receiver to determine the limits of achievable performance. For single user wireless communications the optimal rate and power control strategy to achieve ergodic capacity was determined by Goldsmith and Varaiya [1]. Practical systems represent a different problem in that the set of rates is finite and discrete and there is a tolerable bit error rate (BER) associated with them. Goldsmith and Chua [2] determined the optimal adaptation strategy for such systems using a set of MQAM constellations subject to an instantaneous BER constraint. The optimal adaptation strategy with a given set of rates requires choosing the optimal channel fade thresholds at which the user switches from one constellation to another. These thresholds divide the channel fade space into optimal rate regions. Since the transmit power is a function of just the required rate and the channel fade, power adaptation is fixed once the optimum rate adaptation is determined. Also it was shown in [2] that with just five or six different signal constellations the optimal adaptation strategy achieves within 1-2 dB of the maximum spectral efficiency.

For the multiple access wireless channel the problem gets more interesting as users can interfere with each other. The optimal rate and power adaptation strategy to maximize the ergodic capacity for this channel was determined recently by Tse and Hanly [3]. Knopp and Humblet [7] found that for symmetric users the optimum rate and power adaptation allows only the user with the best channel to transmit at any one time. However optimal rate and power adaptation for interference limited systems when each user has an instantaneous BER constraint and a fixed set of transmit rates to choose from has not been studied. This problem forms the focus of our work in this paper. We concentrate upon multirate DS-CDMA systems since they dominate the air interface standards for third generation wireless communications.

The organization of this paper is as follows. The system model and notations are introduced in Section 2. The general form of the optimal adaptation strategy is presented in Section 3. Section 4 applies the results to particular multirate DS-CDMA systems and presents simple examples to illustrate the nature of the optimal adaptation. Section 5 shows that as the users move around within the cell, i.e. as the propagation path loss and the distribution of the channel fading of each user changes, the optimal power and rate control strategy can adapt by simply scaling the channel fades of the users. We conclude in Section 6 with some observations and further discussion on the interesting aspects of the optimal adaptation strategy and provide directions for 
future work.

\section{System model and notation}

We use the following notation throughout the paper: $E[$. for expectation; overline $(\bar{v})$ for vectors; subscripts $\left(v_{i}\right)$ for the components of the vectors; superscripts $\left(\bar{v}^{2}, \bar{v}^{2}\right)$ to distinguish vectors; $\langle\ldots,$.$\rangle for inner product of vectors;$ $\bar{v}^{1} \circ \bar{v}^{2}$ for the Hadamard product of vectors $\bar{v}^{1}, \bar{v}^{2}$; and $\bar{v}^{(-1)}$ for the Hadamard reciprocal. Optimal solutions are denoted by a ()*

We consider the uplink in a single cell CDMA system with $K$ users, each having a set of $M+1$ transmit rates $\mathcal{M}=\left\{m_{0}, m_{1}, \cdots, m_{M}\right\}$ to choose from, where $m_{0}<$ $m_{1}<\cdots<m_{M}$. The different rates may correspond to different numbers of transmitted codes (multicode CDMA), different bit durations (variable sequence length CDMA), or different modulations (multirate modulation).

An allocation of rates among users is denoted by a rate vector $\bar{n}=\left\{n_{0}, n_{1}, \cdots, n_{K-1}\right\}$. If the system allows users to switch off transmission, i.e. $m_{0}=0$, one or more of these $n_{i}$ may be zero. The system may not allow some rate vectors that are particularly biased towards a subset of users in the interest of fairness, while some rate vectors might be infeasible. Note that a rate allocation is infeasible only if there does not exist a set of non-negative transmit powers that can achieve that rate allocation. The feasibility constraint will be specified more concretely in Section 4 . Assuming $T$ such vectors, the set of allowed and feasible rate vectors in the system is denoted as $\mathcal{N}=\left\{\bar{n}^{0}, \bar{n}^{1}, \cdots, \bar{n}^{T-1}\right\}$ where $T \leq K^{M+1}$.

The user's channel access is assumed to be asynchronous and the spreading codes used by different users are not orthogonal to each other. The channel is affected by slow fading, additive white Gaussian noise (AWGN), and multiple access interference (MAI) due to other users. We define the channel state by the vector $\bar{\chi}=\left(\chi_{1}, \chi_{2}, \cdots, \chi_{K}\right)$ where $\chi_{i}$ is the channel power fade experienced by the $i^{\text {th }}$ user. Under centralized control the optimal transmit rate and power for each user is a function of the entire channel state vector $\bar{\chi}$ which is distributed according to the probability density function $f(\bar{\chi})$. We assume that the receiver (base station) is able to estimate the channel state perfectly and a reliable feedback channel exists from the receiver to each of the transmitters for flow of rate and power control information. The channel state changes at a rate slow enough (slow fading) for the delay on the feedback channel to be negligible. The $i^{\text {th }}$ user's received power at the base station is given by: $P_{i}(\bar{\chi})=S_{i}(\bar{\chi}) \chi_{i} \forall i \in I$ and $\forall \bar{\chi} \in \Gamma$. Here $S_{i}(\bar{\chi})$ is the transmit power of user $i$; $I$ is the index set of users $\{1,2, \cdots, K\}$ and $\Gamma=\mathbb{R}_{+}^{K}$. The $\chi_{i}$ can be the channel power gains themselves (flat fading) or the sum of channel power gains of the multipath components for each user at the output of an L-finger RAKE receiver (frequency selective fading): $\chi_{i}=\sum_{j=1}^{L} \chi_{i, j}$. Using the Hadamard product and Hadamard reciprocal notation we can write $\bar{S}(\bar{\chi})=\bar{P}(\bar{\chi}) \circ \bar{\chi}^{(-1)}$. For MAI we make the Gaussian approximation [6] which has been shown [5] to be valid for large number of users or large enough spreading code lengths.

\section{Optimal adaptation}

The problem statement is as follows:

$$
\begin{array}{r}
\max _{\mathcal{R}_{\bar{n}}} \sum_{i=0}^{T-1} \int_{\mathcal{R}_{\bar{n}^{i}}}<\bar{w}, \bar{n}^{i}(\bar{\chi})>f(\bar{\chi}) d \bar{\chi} \\
\text { s.t. } \int_{\mathcal{R}_{\bar{n}^{i}}} \bar{P}\left(\bar{n}^{i}\right) \circ \bar{\chi}^{(-1)} f(\bar{\chi}) d \bar{\chi} \leq \bar{S}_{a v} \quad \forall i \in I .
\end{array}
$$

The set of $T$ allowed rate vectors $\bar{n}_{i} \in \mathcal{N}$ divides the channel state space into $T$ rate regions $\mathcal{R}_{\bar{n}^{i}}=\left\{\bar{\chi}: \bar{n}^{*}(\bar{\chi})=\right.$ $\left.\bar{n}^{i}\right\}, \forall \bar{n}^{i} \in \mathcal{N}$. The conventional receiver makes the system interference limited. Thus for each allowed rate vector $\bar{n} \in \mathcal{N}$ there is a unique received power vector $\bar{P}(\bar{n})$ that minimizes each user's transmit power for that rate vector and the given set of BER constraints. Note the similarity with the single user adaptive MQAM problem solved in [2]. $\bar{w}$ is the vector of weights or utilities. Constructing the Lagrangian yields the optimal adaptation strategy as follows:

$$
\begin{aligned}
& \bar{n}^{*}(\bar{\chi})=\arg \max _{\bar{n} \in \mathcal{N}}\left(<\bar{w}, \bar{n}>-<\bar{\lambda}, \bar{P}(\bar{n}) \circ \bar{\chi}^{(-1)}>\right) \\
& \bar{S}^{*}(\bar{\chi})=\bar{P}\left(\bar{n}^{*}(\bar{\chi})\right) \circ \bar{\chi}^{(-1)} .
\end{aligned}
$$

$\bar{\lambda}$ is the vector of Lagrange multipliers and needs to be chosen to meet the average transmit power constraints of all users. Note that the equations for the boundaries between optimal rate regions are linear in $\bar{\chi}^{(-1)}$. So the optimal rate regions are polyhedral sets in the Hadamard reciprocal $\bar{\chi}^{(-1)}$ space, and a compact polyhedral set representation exists for $\mathcal{R}_{\bar{n}}$ as:

$$
\mathcal{R}_{\bar{n}}=\left\{\bar{\chi}: A \bar{\chi}^{(-1)} \geq v, \bar{\chi} \in \mathbb{R}_{+}^{K}\right\} .
$$

Here $A=\left\{a_{j, k}\right\}$ is a matrix with $a_{j, k}=\lambda_{k}\left[P_{k}\left(\bar{n}^{j}\right)-\right.$ $\left.P_{k}(\bar{n})\right]$ and $v_{j}=<\bar{w}, \bar{n}^{j}-\bar{n}>$.

Our optimization problem differs from the optimal strategy to achieve capacity in [3], where stronger users can actually be decoded first and their effect cancelled out. Unlike our system which is interference limited, as pointed out in [4], for the capacity maximization problem there is no solution that minimizes the required power of all users. Increasing the power of one user benefits the others because it can be decoded and cancelled more easily.

The optimal rate and power adaptation strategy for our system depends on the probability distributions of the user's 
channel fades $p\left(\chi_{i}\right)$ and the user's average power constraints only through the constants $\bar{\lambda}$. This characteristic is common to the solutions of the problems in [1], [2] and [3]. The similarity between the problems and the nature of their solutions is easily noticeable inspite of the differences pointed out earlier.

\section{Application to specific systems}

While the formula for the optimum adaptation strategy in (1) is sufficiently general, it does not give much insight into the optimal policy for any specific system. In this section we apply (1) to systems using multiple codes or variable spreading - the two commonly used schemes to achieve multiple rates with CDMA. With multicode CDMA, each user has a set of $M$ codes, orthogonal within themselves but not across different users. Variable spreading allows each user $M$ different values of spreading gain while keeping the chip rate fixed. For our example we let the available rates be $\{0,1, \cdots, M\}$, i.e. $m_{i}=i$.

\subsection{Analysis}

For our system model the $i^{\text {th }}$ user's BER with multicode or variable spreading CDMA is given by the following expression:

$$
\begin{aligned}
& \text { if } n_{i}(\bar{\chi}) \neq 0 \text { then } \operatorname{BER}_{i}(\bar{P}(\bar{\chi}), \bar{n}(\bar{\chi}))= \\
& Q\left(\sqrt{\frac{\frac{P_{i}(\overline{)})}{n_{i}(\bar{X})}}{\frac{1}{3 N_{s}} \Sigma_{j \in I-\{i\}} P_{j}(\bar{\chi})+\frac{N_{0}}{2 N_{s} T_{c}}}}\right) \quad \forall i \in I .
\end{aligned}
$$

For multicode CDMA or conventional $\mathrm{CDMA}^{1} N_{s}$ is the spreading gain and $n_{i}(\bar{\chi})$ is the number of orthogonal, synchronous codes transmitted in parallel by user $i$. For notational convenience let us define constants $c=\frac{3 N_{0}}{2 T_{c}}$ and $d=\frac{\left[Q^{-1}\left(B E R_{0}\right)\right]^{2}}{3 N_{s}}$. For variable spreading, $N_{s}$ is the maximum spreading gain (corresponding to the minimum or unit rate) of the system and rates $n_{i}(\bar{\chi})$ are achieved by reducing the spreading factor for user $i$ to $\frac{N_{b}}{n_{i}(\bar{\chi})}$. The chip duration $T_{\mathrm{c}}$ remains fixed in all schemes.

For simplicity we assume symmetric users - The symmetry assumption implies that the users have identical instantaneous BER and average transmit power constraints, their channel power fades are independent and identically distributed (i.i.d.) and the weights in the objective function are set to unity. Therefore it suffices to specify the optimal adaptation for the region $\Gamma_{\text {ord }}=\{\bar{\chi}$ :

${ }^{1}$ Note that conventional CDMA is just a special case of multicode CDMA with $n_{i}(\bar{x}) \in\{0,1\}$ $\left.\chi_{1}>\chi_{2} \cdots>\chi_{K}\right\}$ because the solution for any channel state $\bar{\chi}$ can be obtained by a corresponding transposition of the $\chi_{i} s$.

Note that because of the symmetry assumption the average power constraints of all $K$ users are the same and equivalent to an average power constraint on the sum of the transmitted powers within $\Gamma_{\text {ord }}$. This can be stated as follows:

$$
\text { If } \begin{aligned}
\sum_{i=1}^{K} \int_{\Gamma_{\text {ord }}} S_{i}(\bar{\chi}) f(\bar{\chi}) d \bar{\chi} & =\frac{S_{a v}}{(K-1) !} \\
\text { then } \int_{\Gamma} S_{i}(\bar{\chi}) f(\bar{\chi}) d \bar{\chi} & =S_{a v} \forall i \in I .
\end{aligned}
$$

While in general a rate $\operatorname{sum} r=\sum_{i=1}^{K} n_{i}$ can correspond to several different rate vectors representing various distributions of the total rate among the users, we find that within $\Gamma_{\text {ord }}$ only one of these rate vectors is optimal in that it minimizes the sum transmit power required to achieve a total rate $r$. As pointed out earlier, within $\Gamma_{\text {ord }}$ the average sum power constraint by itself is precisely equivalent to all the user's individual average power constraints over the entire channel fade space $\Gamma$. The unique optimal rate vector $\bar{n}$ is such that $n_{j}=M$ for $j \leq\left\lfloor\frac{r}{M}\right\rfloor, n_{\left\lfloor\frac{r}{M}\right\rfloor+1}=r \bmod M$ and $n_{j}=0$ for $\left\lfloor\frac{r}{M}\right\rfloor+1<j \leq K$. In other words it has the first $\left\lfloor\frac{r}{M}\right\rfloor$ users (with the best channels) transmitting at maximum rate (transmitting their full quota of $M$ codes, or at the minimum spreading gain $\frac{N_{0}}{M}$ ) and one user (the next one) transmitting at rate $m=r \bmod M$ (transmitting $m$ codes or at a spreading gain $\frac{N_{o}}{m}$ ). For simplicity, since each sum rate $r$ corresponds to a unique and distinct rate vector within $\Gamma_{\text {ord }}$, we denote the rate regions as $\mathcal{R}_{\tau}$ instead of $\mathcal{R}_{\bar{n}}$.

The received power vector for rate region $\mathcal{R}_{r}$ is found from (3) so that

$P_{j}(r)= \begin{cases}c \frac{M d}{M d+1} \frac{1}{1-\left\lfloor\frac{r}{M}\right\rfloor\left(\frac{M d}{M d+1}\right)-\frac{M d}{M d+1}} & 0 \leq j \leq\left\lfloor\frac{r}{M}\right\rfloor \\ c \frac{m d}{m d+1} \frac{1}{1-\left\lfloor\frac{r}{M}\right\rfloor\left(\frac{M d}{M d+1}\right)-\frac{m d}{m d+1}} & j=\left\lfloor\frac{r}{M}\right\rfloor+1 \\ 0 & \text { else }\end{cases}$

where $m=r \bmod M$. Note that for a sum rate $r$ to be feasible, i.e. for the transmit powers to be non-negative we need

$$
\left\lfloor\frac{r}{M}\right\rfloor\left(\frac{M d}{M d+1}\right)-\frac{m d}{m d+1} \leq 1 .
$$

Using the optimal adaptation strategy the boundary between the rate regions $\mathcal{R}_{r}$ and $\mathcal{R}_{r+1}$ is given by

$$
\begin{gathered}
\mathcal{R}_{r} \leftrightarrow \mathcal{R}_{r+1}:\left(P_{\left\lfloor\frac{r}{m}\right\rfloor}(r+1)-P_{\left\lfloor\frac{r}{M}\right\rfloor}(r)\right) \Sigma_{j=1}^{\left\lfloor\frac{r}{M}\right\rfloor} \frac{1}{\chi_{j}}+ \\
\left(P_{\left\lfloor\frac{r}{M}\right\rfloor+1}(r+1)-P_{\left\lfloor\frac{r}{M}\right\rfloor+1}(r)\right) \frac{1}{\chi_{\left\lfloor\frac{r}{M}\right\rfloor+1}}=\frac{P_{1}(M)}{\lambda}
\end{gathered}
$$


where $\lambda$ is a constant chosen to satisfy the average power constraint of all users and $\chi_{1}=\lambda$ is the equation for the boundary between $\mathcal{R}_{0}$ and $\mathcal{R}_{M}$.

It is interesting to note that the rate regions $\mathcal{R}_{1}, \mathcal{R}_{2}, \cdots, \mathcal{R}_{M-1}$ that correspond to just one transmitting user with no interference from other users are empty. Mathematically it is easy to see this since the boundary $\mathcal{R}_{0} \leftrightarrow \mathcal{R}_{1}$ is the same as that of $\mathcal{R}_{1} \leftrightarrow \mathcal{R}_{2}, \cdots, \mathcal{R}_{M-1} \leftrightarrow \mathcal{R}_{M}$. In other words the user with the best channel either does not transmit $\left(n_{1}(\bar{\chi})=0\right.$ ) or he transmits at his maximum rate $\left(n_{1}(\bar{\chi})=M\right)$. This suggests that when a user does not have to deal with interference his power is more efficiently used if he transmits only when the channel is good enough for him to transmit at maximum rate. However when more than one user is transmitting, if one of them increases his rate he causes more interference to the others who are forced to increase their powers to meet their BER constraint, forcing him in turn to further increase his power. It no longer turns out to be efficient to wait for a good enough channel to transmit at maximum rate. Thus, for example, rate regions $\mathcal{R}_{M+1}, \mathcal{R}_{M+2}, \cdots, \mathcal{R}_{2 M-1}$ are not empty.

In a system where users are required to maintain some minimum rate $\left(m_{0} \neq 0\right)$, a user will always see interference and allowed rate regions won't be empty. Of course with some fading distributions (like Rayleigh fading) one must allow users to shut off transmission $\left(m_{0}=0\right)$ during deep fades or else the average transmit power will be infinite.

\subsection{Simplifications for conventional CDMA with outage}

For the simple case of conventional CDMA with outage the set of rates available to each user is $\{0,1\}$ indicating that a user may or may not transmit depending upon his rate adaptation. The received power $P_{j}(r)=\frac{c d}{1-(r-1) d}$ for $1 \leq j \leq r$ and 0 otherwise. Hence the equation for the rate boundary between regions $\mathcal{R}_{i}$ and $\mathcal{R}_{i+1}$ is

$$
\begin{aligned}
& \mathcal{R}_{i} \leftrightarrow \mathcal{R}_{i+1}: \quad \Sigma_{j=1}^{i} \frac{d}{\chi_{j}}+\frac{1-(i-1) d}{\chi_{i+1}} \sum_{\mathcal{R}_{i+1}}^{\mathcal{R}_{i}} \\
& \lambda(1-i d)(1-(i-1) d)
\end{aligned}
$$

The relational operator $\sum_{\mathcal{R}_{i+1}}^{\mathcal{R}_{i}}$ means that for all points in $\mathcal{R}_{i}$ the relation is >; for all points in $\mathcal{R}_{i+1}$ the relation is <; and all points on the boundary satisfy this with equality. The optimal rate regions are illustrated for a two user case in Figure 1.

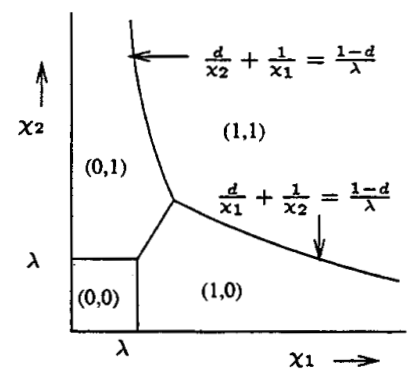

Figure 1. Optimal rate regions for a conventional CDMA system with two users

\subsection{Average throughput computation}

We now present a numerical example to show the application of the optimal adaptation strategy to a multirate CDMA system with symmetric users. We use Monte Carlo simulations to evaluate the average throughput with optimum adaptation, i.e., we generate channel states according to the Rayleigh distribution and find the optimum rate and power given by the adaptation strategy for each state. This is averaged out to obtain the average throughput and transmit power. We used the following parameters: spreading gain $N_{s}=128$, target $\mathrm{BER}\left(\mathrm{BER}_{0}\right)=10^{-3}$ and i.i.d. Rayleigh fading with average channel power fade of unity on each user's channel. We choose noise power spectral density $N_{0}$ and symbol duration $T_{b}$ so that transmitting at unit power all the time would yield an average received $\frac{E_{a}}{N_{0}}$ of $20 \mathrm{~dB}$, i.e. $\frac{T_{b}}{N_{0}}=100$. We evaluate the average sum rate versus average transmit SNR with optimal rate and power adaptation for different values of $M$, the number of codes available to each user. The results are plotted in Figure 2. The plot shows steadily increasing average sum rate for the same average transmit power as $M$ increases.

We also explore the effect of a restricted number of rates available to each user. Figure 3 shows how the average sum rate versus average transmit SNR plot varies as the set of rates available to the user becomes more and more restricted. We notice that when each user is restricted to transmit either at the full rate $(M=16)$ or shut off $(M=0)$ the loss compared to the complete rate set $(M=0,1,2, \cdots, 15,16)$ is less than a $\mathrm{dB}$. Moreover if the rates are restricted to $(M=0,8,16)$ the difference is negligible. The performance penalty depends on the average transmitted SNR and vanishes at high or low average transmit SNRs. To understand this better we plot the optimum rate regions for a system with just 2 users for the case of high average transmitted SNR in Figure 4. The optimum rate regions for low SNRs can be obtained by simply scaling the axes, e.g. contracting the axes by a factor of 7.3 gives us 


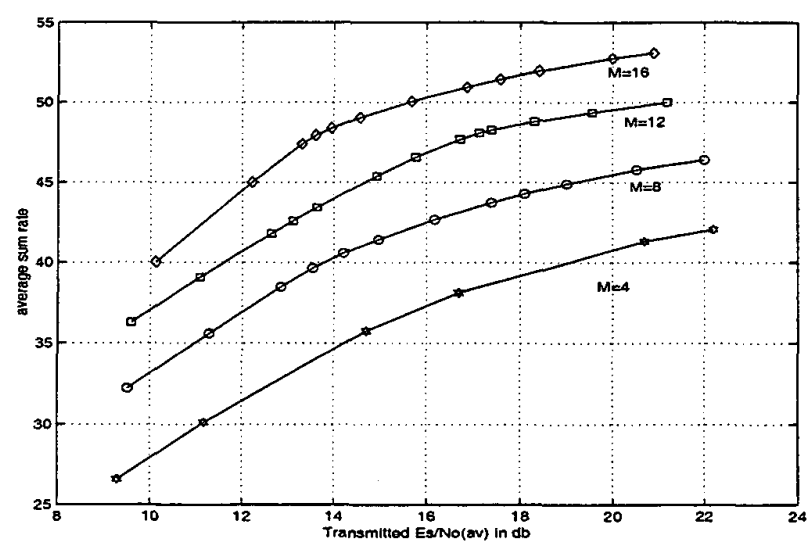

Figure 2. Average Sum Rate versus Average Transmit SNR for 20 users

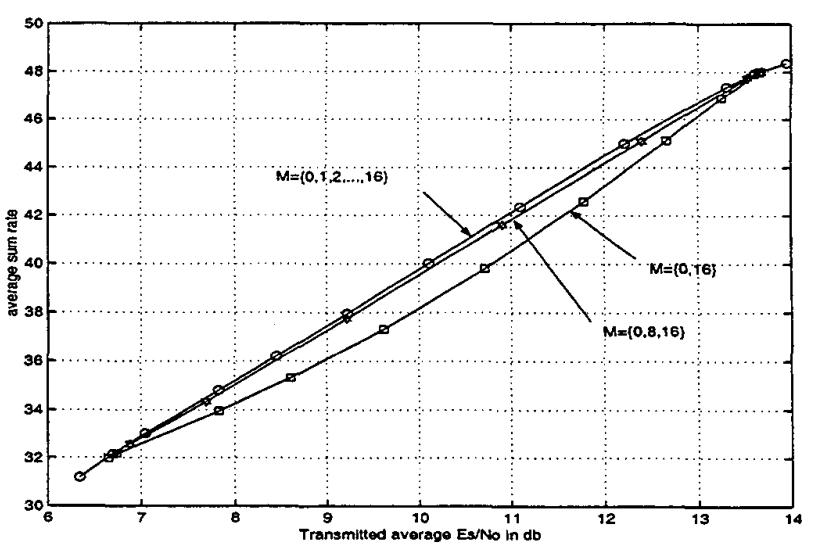

Figure 3. Average Total Sum Rate versus Average Transmit SNR for 20 users

be rewritten as follows:

$\bar{n}^{*}(\bar{\chi})=\arg \max _{\bar{n} \in \mathcal{N}}\left(<\bar{w}, \bar{n}>-<\bar{\lambda} \circ \bar{\chi}^{(-1)}, \bar{P}(\bar{n})>\right)$

Note that the dependence on the $i^{\text {th }}$ user's channel fade $\chi_{i}$ is only through the quantity $\frac{\lambda_{i}}{\chi_{i}}$. Thus a different rate adaptation strategy corresponding to a constant vector $\bar{\lambda}^{1} \neq \bar{\lambda}$ can be treated as effectively the same strategy (i.e. $\bar{\lambda}_{\text {eff }}^{1}=\bar{\lambda}$ ) but with the channel fades scaled by the ratio of the constants (i.e. $\bar{\chi}_{\text {eff }}=\bar{\chi} \circ \bar{\lambda} \circ \bar{\lambda}^{1(-1)}$ ). Thus an appropriate scaling of the channel fades $\bar{\chi}$ (with $\bar{\lambda}$ unchanged) gives the new optimal rate regions. So we could adapt to both smallscale and large-scale mobility. To account for the changing wireless channel power fades $\chi_{i}$ arising out of small scale mobility we have the optimal rate and power adaptation scheme derived earlier. Then in addition, to account for relatively large scale mobility that changes the distribution of the channel fades $p(\bar{\chi})$ itself, we need an algorithm to adapt the scaling factors to retain optimality. The task is made simpler because the algorithm does not have to actually figure out what the new distribution $p(\bar{\chi})$ is or keep track of how it changes. Instead, the algorithm just needs to make sure that every user meets his average transmit power constraint. Each user can evaluate his average transmit power over some time and indicate to the base station if it exceeds or falls short of its desired average transmit power. The algorithm then needs to transform these inputs into a rule that modifies the scaling factors. The apparent simplicity of this adaptation raises some interesting questions about the existence and convergence of efficient algorithms to track the optimal scaling factors as the users move around. It is also interesting to determine the extent of coupling between the effects each of these scaling factors has on the system. 


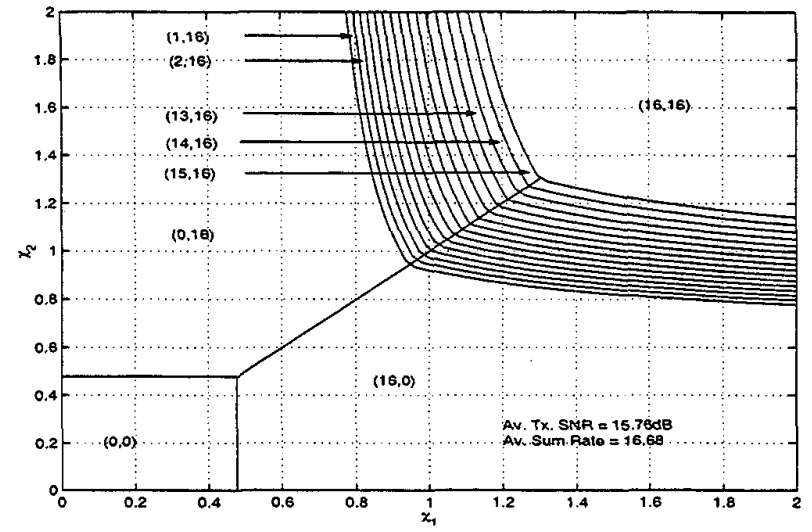

Figure 4. Optimum rate region boundaries for 2 users for high SNR

In other words, how strongly does a change in the constant $\lambda_{i}$ affect the other users' average transmit power as compared to the $i^{\text {th }}$ user's average transmit power. The extent of this coupling should have a significant impact on the nature of such an algorithm as well as its convergence properties. While we do not directly tackle this issue in this paper, we find it interesting enough to include a simple numerical example that leads to some encouraging results.

\subsection{Numerical Example}

We consider the simplest case of 2 users in a conventional DS-CDMA system with Rayleigh fading. However, this time we do not assume symmetry between users. The optimal solution as found earlier depends upon two constants $\lambda_{1}$ and $\lambda_{2}$ to satisfy the users' average transmit power constraints. Define a squared error function as

$$
\mathcal{E}_{S E}=\left(S_{m, a v, 1}-S_{a v, 1}\right)^{2}+\left(S_{m, a v, 2}-S_{a v, 2}\right)^{2}
$$

where $S_{m, a v, 1}$ and $S_{m, a v, 2}$ are the measured average transmit powers of users 1 and 2 respectively. Now varying the constants $\lambda_{1}$ and $\lambda_{2}$, we plot the contours of constant $\mathcal{E}_{S E}$ in Figure 5. Also, in Figure 6 we plot $S_{m, a v, 1}$ against $\lambda_{1}$ keeping $\lambda_{2}$ fixed, for several different values of $\lambda_{2}$. Note that the plots superposed on each other are almost identical and hard to distinguish. Thus $\lambda_{2}$ has little impact on the average transmit power of user 1 . For these plots we used Monte Carlo simulations with the same parameters as Section 4.3.

Figure 5 shows a unique global minimum for the error $\mathcal{E}_{S E}$, suggesting that convergence may not be a big problem for algorithms seeking to minimize $\mathcal{E}_{S E}$. Figure 6 shows that, at least for this simple example, there is almost no

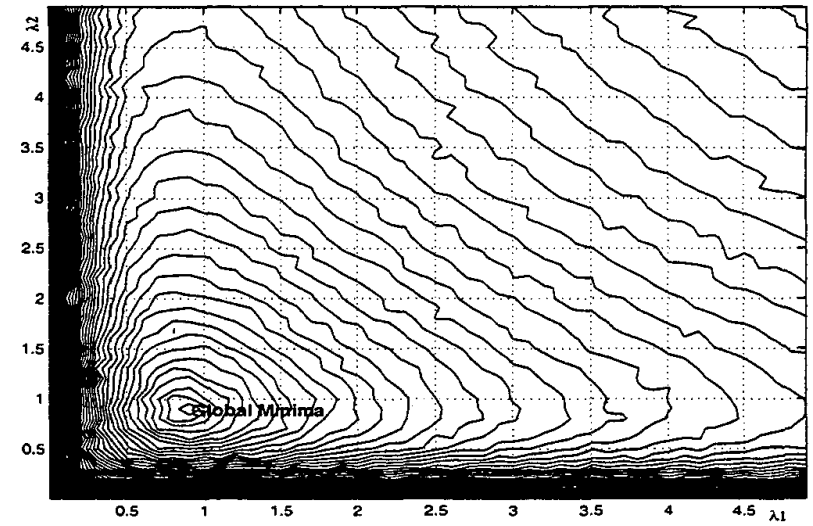

Figure 5. Contour Plots for Squared Error in Measured Average Powers

coupling between the effects of the scaling factors. While $\lambda_{1}$ affects $S_{m, a v, 1}$ very strongly, $\lambda_{2}$ has little effect on it. A possible explanation for this is as follows: For Rayleigh fading the probability mass is heavily concentrated close to zero. So the average transmit power is a strong function of the outage threshold for each user. However, that threshold is a function of that particular constant $\lambda_{1}$ or $\lambda_{2}$ alone for user 1 or 2 respectively. This explains the decoupled effect. Such trends, if proved to be more general than this specific example, could guarantee efficient algorithms with fast convergence.

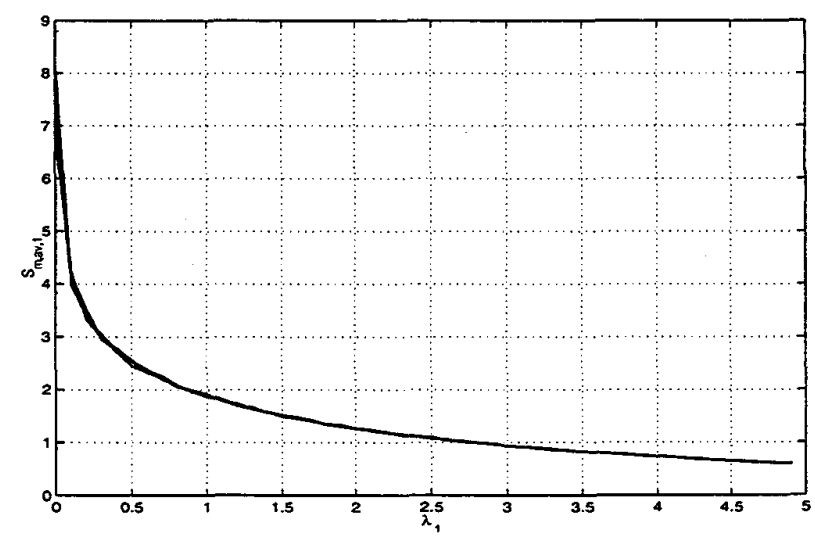

Figure 6. Measured Average Power for user 1 versus $\lambda_{1}$ for various values of $\lambda_{2}$ 


\section{Conclusions and future work}

We determined the optimal centralized adaptive rate and power control strategy to maximize the total average weighted throughput in a generic multirate CDMA system. We found that the optimal adaptation for symmetric users when the set of rates available to the users is discrete and finite allows users to transmit simultaneously and interfere with each other. For multicode and variable spreading CDMA with conventional matched filter detection we found that the user with the best channel either does not transmit (i.e. the best channel is not good enough and nobody transmits) or he transmits at maximum rate if his channel is above a threshold. A user transmits only if all the users with a better channel are transmitting at their maximum rate. Although we considered just the symmetric users case the same holds true for asymmetric users. Our numerical results exploring the effect of granularity of rate adaptation on the average throughput have a similar flavor to corresponding results for a single user in [2] mentioned earlier in Section 1. We found that for a given range of rates available to each user (given the maximum and minimum transmit rates) the optimal average throughput does not improve significantly if we increase the granularity of rate adaptation by allowing each user a richer set of rates. The difference disappears at very low or very high SNRs. Lastly we found that the optimal adaptation strategy has a parametric form where the parameters can be interpreted as scaling factors for the users' channel fades. This allows the optimum adaptation strategy to retain optimality even with large scale mobility by suitably adapting these parameters.

While we focused on multirate DS-CDMA schemes using a conventional receiver, note that the optimal rate and power adaptation strategy given by (1) is sufficiently general to encompass several other systems as well. (1) makes use of the key fact that given a rate vector there exists a unique received power vector that minimizes the transmit power for all the users. This is true for interference limited systems as discussed earlier. In addition the optimal strategy is given by (1) for all other systems where this is true as well. Such systems include a system using a linear MMSE receiver instead of the conventional receiver, and TDMA/FDMA schemes with the users' rates determined by the number of time/frequency slots available to each user.

Some directions for future work include an extension to systems with continuous rates; a detailed analysis of the number of different rates each user must have in the multirate DS-CDMA system to come close to the maximum spectral efficiency; comparison of the optimal adaptation with a fixed-rate scheme that inverts the channel-fading or a truncated power control scheme like the one presented in [8]; and the performance degradation caused by channel measurement errors.

\section{References}

[1] A. J. Goldsmith and Varaiya, P. P., "Capacity of fading channels with channel side information," IEEE Transactions on Information Theory Vol. 43.6, Nov. 1997, Page(s): 1986-1992

[2] A. J. Goldsmith and S. G. Chua, "Variable-Rate Variable-Power MQAM for Fading Channels," IEEE Trans. on Communications, Vol. 45, pp. 12181230, Oct. 1997.

[3] Tse, D.N. and S.V. Hanly (1998) "Multi-access fading channels: Part I: Polymatroid structure, optimal resource allocation and throughput capacities." IEEE Trans. on Info. Theory, v. 44. No. 7, Nov., pp. 27962815.

[4] S.V. Hanly and D. Tse, "Power Control and Capacity of Spread-Spectrum Wireless Networks", Automatica, vol35, no. 12, Dec. 1999, p. 1987-2012.

[5] Tony Ottosson and Ame Svensson, "On Schemes for Multirate Support in DS-CDMA Systems", to be published in Wireless Personal Communications, Kluwer Academic Publishers.

[6] T. S. Rappaport, "Wireless Communications - Principles and Practice", Prentice Hall.

[7] Knopp, R. and P. A. Humblet (1995) 'Information capacity and power control in single-cell multiuser communications'." IEEE Int. Conf. on Communications, Seattle Wash., June.

[8] S.W.Kim and A. Goldsmith, "Truncated power control in code division multiple access communications.", in Vehicular Technology Transactions, May 2000. 